\title{
MaTUTO: adaptação da metodologia de aprendizagem baseada em problemas aplicada ao ensino de ontologias
}

Ricardo Alexandre Afonso, Clarisse Ferrão Pereira

\begin{abstract}
Resumo
Introdução: A implantação de novas metodologias de ensino traz consigo uma proposta de evolução dos modelos tradicionais de ensino, os quais têm se mostrado menos eficientes em decorrência da evolução da sociedade e do uso da tecnologia. Esta inadequação educacional incentivou a construção de uma proposta que condensa as etapas da metodologia de Aprendizagem Baseada em Problemas a qual foi testada na criação de uma ontologia de domínio definida pelo tutor do processo de ensino/aprendizagem. 0 objetivo do artigo é apresentar uma metodologia compatível com uma realidade dinâmica existente em disciplinas e aulas que enfocam sistemas de informação, procedendo a uma avaliação com o grupo de alunos envolvido no experimento. Método: Apresentam-se as principais caracteristicas e etapas da Aprendizagem Baseada em Problemas, as quais foram ajustadas na proposta MaTUTO (Mapear, TUtoriar e Traduzir em Ontologia) aplicada a grupos de alunos de graduação. Resultados: Foi possivel desenvolver uma proposta metodológica que adapta e condensa os passos originais da Aprendizagem Baseada em Problemas como apoio às dinâmicas existentes em disciplinas ministradas nos cursos de Sistemas de Informação. A ontologia foi construida a contento e a avaliação do processo oferece resultados animadores. Conclusões: Para a temática indicada - a ontologia de domínio - a proposta metodológica alternativa permitiu aos alunos não apenas a assimilação dos conceitos envolvidos na Aprendizagem Baseada em Problemas, como resultou em um produto de organização da informação, ou seja, a ontologia.
\end{abstract}

\section{Palavras-chave}

Aprendizagem baseada em problemas. Metodologia do ensino. Processo ensino-aprendizagem. Ontologia. Sistemas de informação.

\section{Introdução}

Nos cursos de graduação em informática, e especificamente nos cursos de Sistemas de Informação, a implantação de novos modelos educacionais com características estimulantes e voltadas à participação podem preencher lacunas encontradas no modelo tradicional de ensino, pois remetem os estudantes a buscarem soluções para problemas vivenciais, relacionando teoria e prática. Freire (2001, p. 25) considerava que o educador precisa saber que "ensinar não é transferir conhecimento, mas criar as possibilidades para a sua produção ou a sua construção". Esta afirmativa se contrapõe aos modelos tradicionais de ensino utilizados pelas áreas de exatas, onde o mestre, respaldado por seus anos de experiência em áreas específicas, transmite este saber em aulas expositivas e em alguns casos, com baixo grau de interação.

A limitação desta proposta tradicional, desafiada pelas inovações crescentes, fez com que a comunidade científica pesquisasse sem alternativas de novos processos de ensino-aprendizagem, capazes de formar futuros engenheiros com visão holística ao integrar a ciência com a prática e estimulando o aprendizado ativo e baseado em competências.

Ao analisar modelos educacionais, (BRANSON, 1990 apud COSTA-PINTO, 2001) propôs uma alternativa que reconhece a evolução do paradigma educacional apoiada na utilização de compu- 
tadores. $\mathrm{O}$ autor afirma que, no passado, a figura do professor se caracterizava como transmissor e único detentor do conhecimento, e ao estudante, cabe o papel de receptor. No presente, mesmo com uma relação mais próxima entre o estudante e o professor, este ainda continua a ser a única fonte de conhecimento e de experiência.

Sob outro enfoque, estudante e professor, em um modelo centrado em tecnologia e voltado à implantação de sistemas especialistas ou mesmo ontologias - como o relatado nesse trabalho - volta o foco para a, interação entre estes agentes, tendo a tecnologia como facilitadora desse fluxo (Figura 1$)$.

Figura 1 - Evolução histórica do paradigma de ensino

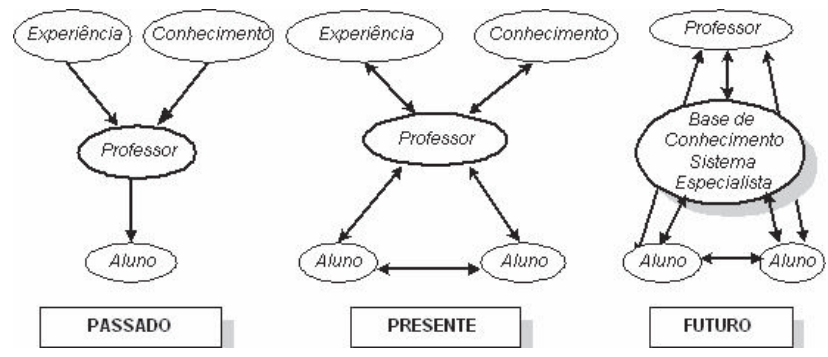

Fonte: adaptado de Freitas (2001).

Criar uma metodologia compatível com uma realidade dinâmica existente em disciplinas e aulas relativas à sistemas de informação é um dos desafios apresentados neste trabalho. Indo ao encontro do uso de uma metodologia de ensino moderna, combinada com recursos tecnológicos tangentes que possam juntos viabilizar o processo de transferência e compartilhamento de conhecimento aos alunos em um domínio específico, a presente investigação apresenta o resultado dessa sinergia à qual foi atribuído o nome MaTUTO (Mapear, TUtoriar e Transformar em Ontologia).

Para alcançar os objetivos propostos, este trabalho descreve a relação entre a Aprendizagem Baseada em Problemas e a estratégia de ensino-aprendizagem. Em seguida, apresenta-se a ontologias de domínio como temática para a estratégia proposta, a aplicação desta no contexto de sala de aula, seguida da avaliação da experiência pelos alunos e aspectos complementares, a guisa de conclusão.

\section{Aprendizagem Baseada em Problemas como estratégia de ensino- aprendizagem}

Segundo Gil (2006), a Aprendizagem Baseada em Problemas (ABP) é uma estratégia onde os estudantes trabalham com o objetivo de solucionar problemas através de estudos de caso previamente montados.

Neste modelo, o estudante muda de papel no processo de aprendizagem, passando de receptor passivo para ativo, e assumindo responsabilidade pelo seu aprendizado. A ênfase no compromisso e responsabilidade individual é de suprema importância para que os objetivos educacionais sejam alcançados.

Esta nova concepção de ensino foi primeiramente utilizada na área de saúde. Porém, várias áreas a utilizam na sua totalidade ou parcialmente, como por exemplo, o Departamento de Engenharia Mecânica da Pontifícia Universidade Católica do Rio de Janeiro (PUC-Rio) que faz uso de uma metodologia interativa aplicada com utilização de ferramentas tecnológicas (BRAGA, 2002), o Departamento de Pós-graduação de Física da Universidade de São Carlos (RIBEIRO; ESCRIVÃO FILHO; MIZUKAMI, 2003) e o Departamento de Contabilidade da Universidade de São Paulo (USP) (ARAÚJO; RODRIGUES, 2006).

A metodologia $\mathrm{ABP}$ apoia-se em grupos tutoriais, que são compostos por um pequeno agrupamento de estudantes (entre oito e doze), acompanhados de um tutor. Nestes encontros, um problema ou caso pré-estruturado é colocado em discussão e mediado pelo tutor, que orienta seus aprendizes a pensar de forma racional e lógica.

Por tratar, a cada caso, de temáticas distintas, cada sessão de tutoria escolhe, de forma democrática, um coordenador e um secretário. Isso acontece de maneira contínua, pois ao término de um caso um novo tema é proposto e um novo coordenador e um novo secretário são eleitos. 
A dinâmica do grupo tutorial obedece a uma técnica própria denominada de "sete passos" elencada por Schmidt (1993):

a) clarificar os conceitos não compreendidos na leitura do problema;

b) definir o problema;

c) analisar o problema;

d) desenhar um inventário das explicações inferidas a partir do passo " $c$ ";

e) formular objetivos de aprendizagem;

f) coletar informações adicionais fora do grupo; e

g) sintetizar e testar as informações recém-adquiridas.

As avaliações acontecem ao final de cada módulo, com base nos objetivos e conhecimentos específicos definidos no projeto da instituição. Segundo Schmidt (1993), o tutor é fundamental ao bom desenvolvimento das atividades do grupo, tendo como principais atribuições: estimular todos os membros do grupo a participarem das discussões; ajudar o coordenador com a dinâmica do grupo e na administração do tempo; assegurar que as anotações sejam corretamente realizadas pelo secretário; evitar desvios na discussão; assegurar que o grupo atinja, no mínimo, os objetivos de aprendizagem preestabelecidos; checar a compreensão do grupo; e, avaliar o desempenho dos membros e do grupo como um todo. Ao estudante que desempenha a função de coordenador cabe: liderar o grupo durante o processo; estimular todos os membros do grupo para que participem das discussões; manter a dinâmica do grupo; administrar o tempo; assegurar que o grupo cumpra a sua tarefa, assegurar que o secretário acompanhe as discussões e realize corretamente as anotações. $\mathrm{O}$ estudante na função de secretário tem responsabilidades de: anotar os termos desconhecidos, os problemas identificados, as formulações e hipóteses oferecidas e os objetivos de aprendizagem definidos; ajudar o grupo a ordenar as suas ideias; participar das discussões; enviar a primeira fase de anotações aos membros do grupo; anotar as fontes usadas pelo grupo; elaborar o relatório final; e, enviar o relatório final aos membros do grupo. Aos demais estudantes cabe seguir em sequência os sete passos do proces- so; participar das discussões; ouvir com atenção e respeito à contribuição dos demais membros; perguntar abertamente sem receios; pesquisar, no mínimo, todos os objetivos de aprendizagem estabelecidos; partilhar o conhecimento adquirido com os colegas (SCHMIDT, 1993).

A ABP se mostra eficiente no ensino independentemente da área de aplicação e, quando amparada por ferramentas da Tecnologia da informação proporciona interação, integração, busca e recuperação de informações. O dinamismo característico deste método obriga os tutores, estudantes e corpo técnico a uma atitude proativa e de expressão de opinião contínua. Para que as respostas sejam ágeis e atendam as solicitações dos participantes no tempo proposto, o apoio tecnológico também deve ser investigado de forma a dar suporte à formação dos estudantes (PEREIRA, 2006).

\section{Ontologias como temática para a experiência voltada à Aprendizagem Baseada em Problemas}

Historicamente o termo ontologia tem origem no grego onus (ser) e logos (palavra). O termo original é a palavra aristotélica "categoria", que pode ser usada para classificar alguma coisa. Aristóteles apresenta categorias que servem de base para classificar qualquer entidade e introduz ainda o termo "diferente" para propriedades que distinguem distintas espécies do mesmo gênero.

Segundo Guarino (1998), as ontologias são classificadas em diferentes tipos de acordo com seus conteúdos, podendo se dividir em genéricas, de domínio, de tarefas, de aplicação e de representação.

Objetivando construir uma ontologia para ambientes colaborativos e dinâmicos de aprendizagem, propôs-se aos alunos uma solução utilizando OWL-DL (Web Ontology Language) que se trata de uma linguagem para definir e instanciar ontologias na Web baseada em parte nas lógicas de descrição, a qual foi modelada com o apoio da ferramenta Protégé (STANFORD..., 2013). 
Para fins desta experiência se utilizou o termo ontologia sempre em menção à classificação de ontologias de domínio, uma vez que esse conceito representa uma ontologia que possui um vocabulário de conceitos, definições e suas propriedades, e é representada por um modelo formal de relações entre esses conceitos e os axiomas capazes de apresentar de maneira clara e não ambígua o conhecimento do domínio (CHANDRASEKARAN, 1999).

\section{A experiência MaTUTO: Mapear, TUtoriar e Traduzir em Ontologia}

A proposta de construção apoiou-se nos preceitos da ABP, a qual determina que o trabalho seja realizado em grupos tutoriais, constituídos por estudantes e um tutor. Este último pode ser um estudante ou professor que possua o domínio do assunto em questão, ou que tenha conhecimentos suficientes para criar a estrutura semântica inicial da ontologia a ser desenvolvida.

Para adequar a metodologia ABP para a construção da ontologia, os "sete passos" propostos por Schmidt (1993), foram sintetizados em três etapas (Quadro 1).

Para se avaliar o uso da proposta MaTUTO enquanto uma metodologia, esta foi inserida como atividade complementar da disciplina de Enge-

Quadro 1 - Adequação dos passos da APB para a construção das Ontologias

\begin{tabular}{|c|c|c|c|}
\hline \multicolumn{2}{|r|}{$\mathrm{ABP}$} & \multicolumn{2}{|c|}{ MaTuTO } \\
\hline Passo 1 & $\begin{array}{l}\text { Clarificar os } \\
\text { conceitos }\end{array}$ & \multirow{3}{*}{$\begin{array}{c}\text { Etapa1 } \\
(\mathrm{P} 1, \mathrm{P} 2, \mathrm{P} 3)\end{array}$} & \multirow{3}{*}{ Mapear } \\
\hline Passo 2 & Definir o problema & & \\
\hline Passo 3 & Analisar o problema & & \\
\hline Passo 4 & $\begin{array}{l}\text { Desenhar um } \\
\text { inventário das } \\
\text { explicações }\end{array}$ & \multirow{2}{*}{$\begin{array}{c}\text { Etapa } 2 \\
\text { (P4 e P5) }\end{array}$} & \multirow[t]{2}{*}{ TUtoriar } \\
\hline Passo 5 & $\begin{array}{l}\text { Formular objetivos } \\
\text { de aprendizagem }\end{array}$ & & \\
\hline Passo 6 & $\begin{array}{l}\text { Coletar informações } \\
\text { adicionais }\end{array}$ & \multirow{2}{*}{$\begin{array}{l}\text { Etapa } 3 \\
\text { (P6 e P7) }\end{array}$} & \multirow{2}{*}{$\begin{array}{c}\text { Traduzir } \\
\text { em } \\
\text { Ontologia }\end{array}$} \\
\hline Passo 7 & $\begin{array}{l}\text { Sintetizar e testar as } \\
\text { informações }\end{array}$ & & \\
\hline
\end{tabular}

Fonte: adaptado de Schmidt (1993). nharia de Software II do curso de Sistemas de Informação (primeiro semestre de 2008). O experimento atingiu duas turmas de aproximadamente trinta alunos cada.

O cenário proposto apresentou a temática dos testes de software em bancos de dados autônomos como problema a ser discutido pelos alunos. A tutoria ficou a cargo do professor da disciplina (cujo objeto de estudo em sua dissertação de mestrado foi solução de problemas em bancos de dados autônomos com o apoio de ontologias) e, portanto, qualificado para tutorar o processo inicial do mapeamento da ABP para a nova proposta.

A definição detalhada de cada uma das etapas da proposta apresenta-se na sequência, assim como a exemplificação do uso dessas etapas para o problema proposto.

Mapear: conceitos, definição e análise do problema

Os três passos iniciais da $\mathrm{ABP}$, e respectivos conceitos, foram condensados, na proposta MaTUTO, em uma única etapa chamada "Mapear". No exemplo de utilização, coube aos alunos a busca de referências e material bibliográfico suficiente para compreender os conceitos inerentes de bancos de dados orientados a objetos, seus erros e falhas, e a forma como são resolvidos (Figura 2).

Os alunos concluíram que, de acordo com a Internacional Electrotechnical Commission (2013)

Figura 2 - Representação gráfica para Problema, Erro e Falha

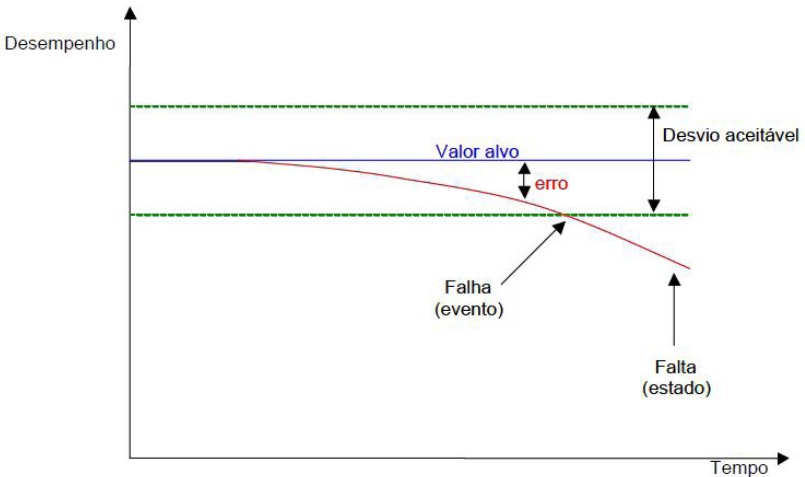

Fonte: adaptado de Rausand e Oien (1996). 
um erro é representado pela discrepância entre um valor (ou condição) medido, observado ou computado, e a verdade (especificada ou teoricamente correta). Assim, é possível afirmar que um erro não necessariamente é uma falha apenas pelo fato de estar dentro de limites aceitáveis de desvio de um desempenho desejado (valor alvo). Sendo assim, uma situação originária de problema só é determinante para que ocorram erros (independente do fator desempenho) e esses por sua vez podem ao longo do tempo causar falhas.

Com base nessa definição, o mapeamento tornou os conceitos mais claros e compreensíveis, assim como a análise das possíveis soluções a serem empregadas no problema. Como exemplo, podese citar o conceito de "erro", que - na ontologia - servirá como classificação para determinar se o problema é recorrente ou ocasional no universo dos Sistemas Gerenciadores de Bancos de Dados (SGBD). Para isso, decidiu-se que este não será instanciado como classe, apenas como uma propriedade transitiva na etapa de tradução do problema na ontologia proposta (Etapa 3).

Aliado a essa compreensão, foi possível classificar tipos de ações (reativas ou proativas) que devem ser tomadas diante destes eventos, uma vez que essa ontologia prevê ações autônomas a serem desempenhadas por sistemas mais modernos em uso.

\section{TUtoriar: inventário das explicações e objetivos de aprendizagem}

Embora a figura do tutor apareça em todas as etapas da ABP apresentada, sua figura aparece em destaque na segunda etapa da proposta MaTUTO, que sintetiza os passos 6 e 7 da metodologia ABP. Esta etapa, na MaTUTO, é nomeada como "TUtoriar".

A figura do tutor nessa etapa se consolida pela absorção das figuras do Coordenador e do Secretario em um só membro do grupo, embora não se exclua a possibilidade de utilizar estudantes para desempenhar esses papeis. No experimento aqui apresentado essa figura não apresentou necessi- dade de disjunção por se tratar de um professor especialista na área de bancos de dados como já mencionado.

O inventário das soluções propostas para os principais problemas ocorrentes em bancos de dados, somado com as interações entre os estudantes serviram como base para que o tutor pudesse definir sua estratégia para atingir os objetivos de aprendizagem. Para isso, a proposta se adaptou aos resultados da escolha estratégica do tutor e das necessidades de resultados buscados pelos estudantes. Porém, não deixou de atender ao preceito de que o objeto de aprendizagem seja composto por conteúdo, prática e avaliação.

Coube ao tutor nesse trabalho criar um mapa de explicações sobre os conceitos de bancos de dados e das principais mensagens de erro e aviso encontrados durante a execução de rotinas nesses bancos de dados. Esse resultado foi divulgado através de arquivos texto contendo os códigos de erro, identificadores e textos explicativos contendo a rotinas de solução para esses problemas.

O passo dedicado a formular os objetivos de conhecimento originário da metodologia ABP, foi incluído na etapa de TUtoriar da proposta MaTUTO e objetivou capacitar o estudante a:

a) identificar os principais conceitos sobre SGBD, suas mensagens de erro e de aviso em determinadas situações;

b) identificar a diferença entre erro, falha e problema e aplicar esses conceitos na solução de problemas ocorridos em bancos de dados; e

c) compreender e escolher quais as melhores soluções para automatizar as rotinas de manutenção desses bancos de dados.

Embora os objetivos dessa etapa tenham sido definidos antes do inicio do trabalho de aquisição de conhecimento, houve a preocupação de avaliar se tais objetivos estavam sendo atingidos durante a execução desse processo. Os resultados dessa avaliação são apresentados na seção final deste trabalho. 


\section{Traduzir em Ontologia: informações} adicionais, síntese e teste

Como tarefa inicial dessa etapa, coube ao tutor criar a estrutura da ontologia e a forma como os problemas poderiam ser ali classificados. Aos estudantes, coube questionar e propor mudanças nessa estrutura a fim de que ela pudesse ser extensível ao maior número de problemas possíveis.

Como resultados na construção da ontologia, a classe "Problema" atendeu aos questionamentos iniciais e sua estrutura original manteve o relacionamento hierárquico com os diferentes tipos de problemas detectados em Sistemas Gerenciadores de Bancos de Dados (SGBD). A Figura 3 apresenta um exemplo de um problema relacionado a fragmentação de índices, que é classificado como um erro recorrente e que ao longo do tempo pode dar origem a uma falha por impedir que o sistema execute rotinas que utilizem tais índices.

Figura 3 - Exemplo de um problema em Sistema Gerenciador de Base de Dados

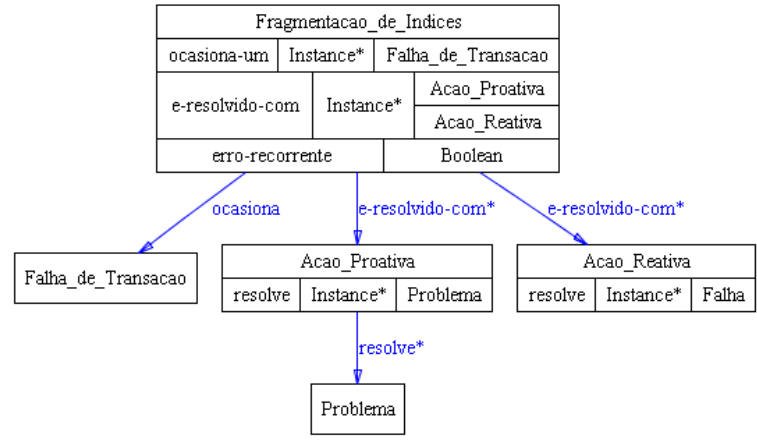

Fonte: dados da pesquisa (2008).

Após definida a estrutura da ontologia de domínio e definido o escopo que seria atendido pelas classes Problema e Falha, os estudantes colaboraram com as definições sobre tipos de problemas e falhas a serem classificados. A Figura 4 apresenta as classes anteriormente citadas, e as adições semânticas sugeridas pelos estudantes.
Figura 4 - Definição semântica de tipos de problemas e falhas em SGBD

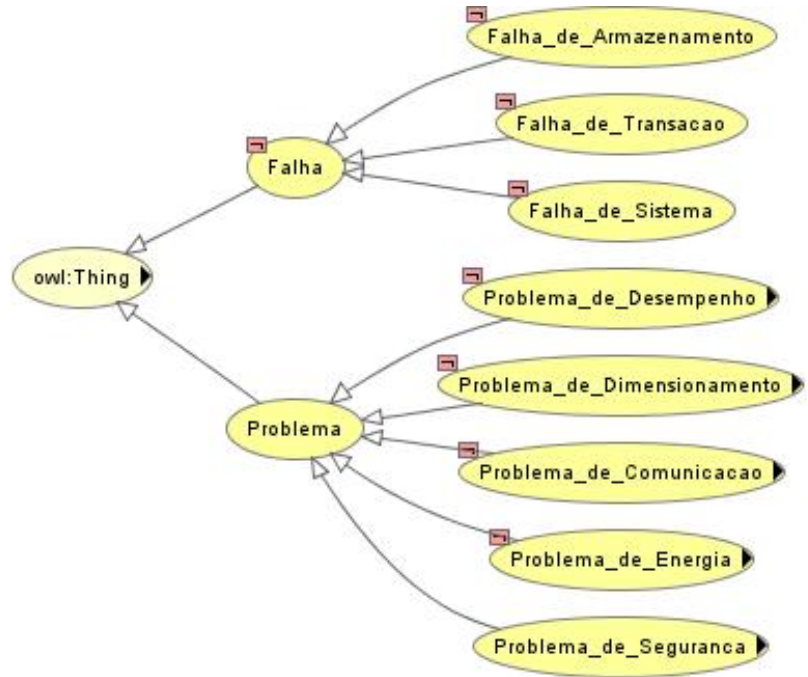

Fonte: dados da pesquisa (2008).

A etapa final de tradução da estratégia em ontologia de domínio resultou na criação de uma ontologia formal dividida em quatro classes principais, chamadas de superclasses, que são: "Falha", "Ação", "Problema" e "Tarefa" (Figura 5).

Figura 5 - Relação entre as classes da Ontologia de Domínio

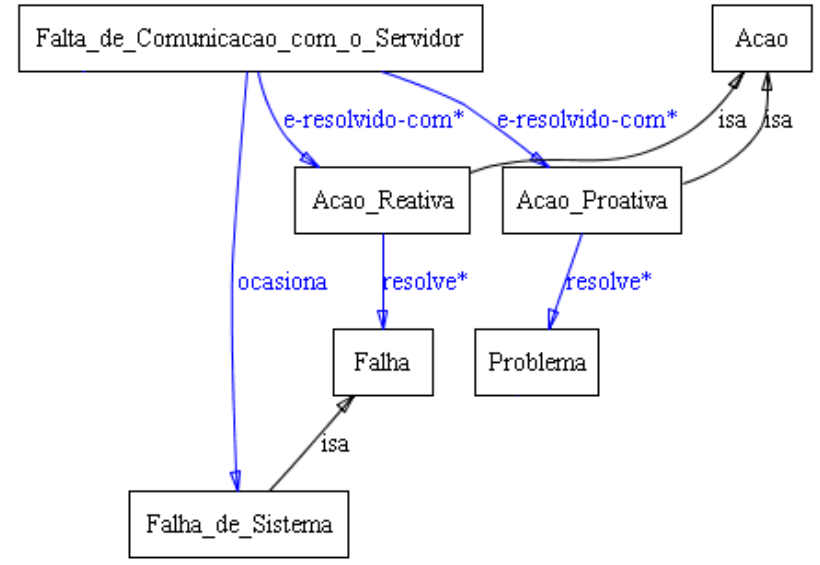

Fonte: dados da pesquisa (2008).

Na etapa 3, definida como etapa de Tradução, o tutor interagiu com os estudantes para a coleta de novas informações voltadas à realização de testes exaustivos da ontologia proposta. Uma forma bastante comum de validar ontologias consiste na criação das restrições. Os estudantes criaram algumas restrições com base em Breitman (2004), que esclarece que - em OWL-DL - as restrições 
são utilizadas para circunscrever os indivíduos de uma classe. Nesse exemplo, o quantificador universal ${ }^{1}$ utilizado foi: "é-classificado-como" e o quantificador existencial ${ }^{2}$ foi: "esta-associado".

Após este ciclo se verificou que a proposta MaTUTO atendeu as etapas pré-definidas para o experimento, encerrando-se com a criação da ontologia onde os casos de erro, falha e problemas puderam ser catalogados e inseridos como itens dessa ontologia.

\section{Conclusões}

O objetivo deste trabalho foi de apresentar um caso prático da utilização de uma proposta metodológica capaz de condensar os passos tradicionais da ABP em etapas distintas que possam atender às características dinâmicas de disciplinas ministradas nos cursos de Sistemas de Informação.

Durante a utilização da metodologia, criação e testes da ontologia proposta, 54 alunos foram submetidos a três avaliações simples de desempenho para avaliar o nível de satisfação com a nova forma de aquisição de conhecimento. Estas avaliações ocorreram em 10/03/2008 (inicio do semestre letivo - A), 07/04/2008 (data que antecedeu as primeiras avaliações semestrais - B) e 05/05/2008 (período pós-avaliações C) (Figura 6). A avaliação consistiu em três perguntas, a saber:

a) domina os conceitos apresentados? (Azul)

b) é capaz de definir uma ontologia? (Vermelho) e

c) a metodologia MaTUTO é confiável? (Verde)
Figura 6 - Gráfico de avaliação de aquisição de conhecimento com o uso da proposta metodológica MaTUTO

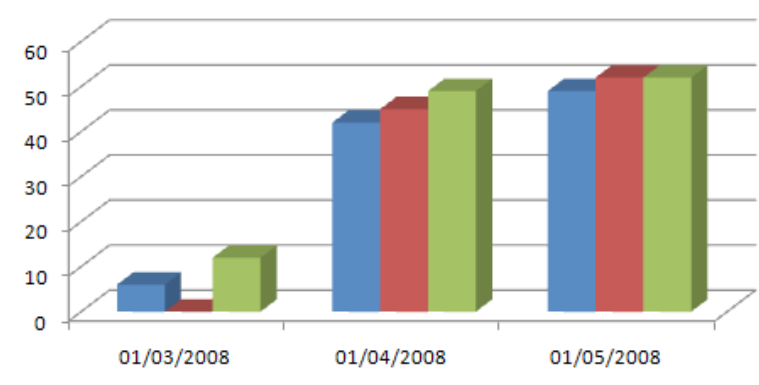

A

B

C

Fonte: dados da pesquisa (2008).

A metodologia MaTUTO, ao se basear nos preceitos da ABP, apresentou resultados satisfatórios no que diz respeito à interação entre tutor e estudantes. O objetivo primário (criação de uma ontologia) foi atingido e foi possível satisfazer as condições para a transmissão e compartilhamento do conhecimento no grupo e com o tutor.

A metodologia ABP vem sendo utilizada em algumas universidades brasileiras (SANTOS, 2007), o que demonstra interesse da comunidade acadêmica na expansão do uso do método para áreas que não estejam diretamente ligadas a área de saúde, pioneira no seu uso.

Entre as possibilidades de continuidade da experiência, trabalhos futuros podem aprofundar as adequações da metodologia ABP direcionando-a para cursos à distância nas áreas de Ciências Exatas, por exemplo, explorando particularidades desta modalidade de ensino e sua aplicação prática.

\footnotetext{
${ }^{1}$ o quantificador universal (usualmente $\forall$ ) é o símbolo usado para denotar o universo de quantificação, informalmente lido como "para todo".

${ }^{2}$ o operador lógico $\exists$ é usado para denotar a quantificação existencial, que representa a predicação de uma propriedade ou relação para, pelo menos, um elemento do domínio.
} 


\section{Referências}

ARAÚJO, A. M. P. O., RODRIGUES, E. de. A. Ensino da Contabilidade: aplicação do método PBL nas disciplinas de contabilidade em uma Instituição de Ensino Superior Particular. In: CONGRESSO DE CONTROLADORIA E FINANÇAS, 6., São Paulo, 2006. Anais eletrônicos... USP, São Paulo. Disponível em: <http://www.congressousp.fipecafi.org/ artigos62006/448.pdf >. Acesso em: 25 maio 2013.

BRAGA, W. Evaluating students on Internet Enhanced Engineering Courses. In: ASEE/IEEE FRONTIERS IN EDUCATION, 32., Boston, MA, USA, 2002. Disponível em: <http://wwwusers.rdc.puc-rio.br/ wbraga/transcal/pdf/Papers/FIE02.pdf $>$. Acesso em: 25 maio 2013.

BRANSON, R. Issues in the design of schooling: changing the paradigm. Educational Technology, v. 30, n. 4, p. 7-10, Apr. 1990.

BREITMAN, K. K. Web semântica: a internet do futuro. Rio de Janeiro: LTC, 2005.

CHANDRASEKARAN, B. What are ontologies, and why do we need them? IEEE Inteligent Systems, v. 14, n. 1, p. 20-25, Jan. 1999. Disponível em: <http://www.csee. umbc.edu/courses/771/papers/chandrasekaranetal99. pdf>. Acesso em: 25 maio 2013.

\section{COSTA-PINTO, S. D. N. C. O computador e o ensino} superior de matemática: uma prática interativa. Dissertação (Mestrado em Engenharia da Produção) - Universidade Federal de Santa Catarina, 2001. Florianópolis, 2001.

FREIRE, P. Educação e mudança. 24. ed. São Paulo: Paz e Terra, 2001.

FREITAS, W. F. Utilização de tecnologia de groupware no desenvolvimento de recursos humanos: uma análise comparativa entre dinâmicas disjuntas no ambiente de trabalho da Prefeitura Municipal de Belo Horizonte. Belo Horizonte: Fundação João Pinto, 2001.

GIL, A. C. Didática do ensino superior. São Paulo: Atlas, 2006.

GUARINO, N. Formal ontology in information systems. In: FORMAL ONTOLOGY IN INFORMATION SYSTEMS CONFERENCE, 1., Trento, Itália, 1998. Proceedings... Amsterdam: IOS, 1998. Disponível em: $<$ http://uosis.mif.vu.lt/ donatas/Vadovavimas/Temos/ OntologiskaiTeisingasKoncepcinisModeliavimas/ papildoma/Guarino98-Formal\%20Ontology\%20 and\%20Information\%20Systems.pdf $>$. Acesso em: 25 maio 2013.
INTERNATIONAL ELECTROTECHNICAL

COMMISSION. Electropedia: the world's online electrotechnical vocabular. 2013. Disponível em: $<$ http://www.electropedia.org/>. Acesso em: 25 maio 2013.

PEREIRA, C. F. Tecnologia da informação utilizada como suporte ao ensino médico de graduação na Aprendizagem baseada em problemas (ABP). 2006. Dissertação (Mestrado) - Universidade Federal de Pernambuco, Recife, 2006. Disponível em: <http://goo. gl/TwZZM>. Acesso em: 25 maio 2013.

RAUSAND, M.; ØIEN, K. The basic concepts of failure analysis. Reliability Engineering and System Safety, v. 53, n. 1, p. 73-83, Jun. 1996. Disponível em: <http:// dx.doi.org/10.1016/0951-8320(96)00010-5>. Acesso em: 25 maio 2013.

RIBEIRO, L. R. C.; ESCRIVÃO FILHO, E.; MIZUKAMI, Ma. da G. N. Uma experiência com a PBL no ensino de engenharia sob a ótica dos alunos. In: CONGRESSO BRASILEIRO DE EDUCAÇÃO EM ENGENHARIA, 31., Rio de Janeiro, 2003. Anais eletrônicos... Rio de Janeiro: ABENGE, 2003. Disponível em: <http:// www.abenge.org.br/CobengeAnteriores/2003/artigos/ NMT221.pdf>. Acesso em: 25 maio 2013.

SANTOS, D. M. B. dos; PINTO, G. R. P. R.; SENA; C. P. C.; BERTONI, F. C.; BITTENCOURT, R. A. Aplicação do método de Aprendizagem Baseada em Problemas no curso de Engenharia de Computação da Universidade Estadual de Feira de Santana. In: CONGRESSO BRASILEIRO DE EDUCAÇÃO EM ENGENHARIA, 35., 2007, Curitiba. Anais eletrônicos... Curitiba: Centro Universitário Positivo, 2007. p. 2A07-12A07-14. Disponível em: <http://www.abenge.org.br/ CobengeAnteriores/2007/artigos/286-Gabriela\%20 Ribeiro\%20Peixoto\%20Rezende\%20Pinto.pdf $>$. Acesso em: 20 jun. 2013.

SCHMIDT H.G. Foundations of problem-based learning: some explanatory notes. Medical Education, v. 27, n. 5, p. 422-432, 1993. Disponível em: <http://repub.eur. $\mathrm{nl} / \mathrm{res} /$ pub/2709/eur_schmidt_107.pdf $>$. Acesso em: 25 maio 2013

\section{STANFORD CENTER FOR BIOMEDICAL} INFORMATICS RESEARCH. Protégé Overview. Disponível em: <http://protege.stanford.edu/ overview/>. Acesso em: 25 maio 2013.

\section{Agradecimentos:}

Este trabalho foi parcialmente financiado pela Fundação de Amparo à Ciência e Tecnologia do Estado de Pernambuco (FACEPE), concessão IBPG-0499-1.03/11. 
MaTUTO: a problem-based learning approach applied to an ontology teaching case

\section{Abstract}

Introduction: The introduction of new teaching methodologies brings a proposal for the evolution of traditional teaching models, which have been shown to be less efficient due to the evolution of society and the use of technology. This inadequacy educational encouraged the construction of a proposal that condenses the steps of the Problem-Based Learning methodology, which was tested in the creation of a domain ontology defined by a tutor responsible for the teaching/ learning activity. The article aims to present a methodology compatible with a dynamic reality that takes place at information systems disciplines and classes. The students involved in the experiment were also invited to evaluate the experience. Method: It presents the main features and steps of the Problem-Based Learning methodology, adjusted in the proposed MaTUTO (Map, TUtoring and Translate in Ontology), which was applied to undergraduate students. Results: It was possible to apply a methodology that adapts and condenses the original steps of the Problem-Based Learning approach as a support to subjects taught at Information Systems courses. The ontology was built and found satisfactory, as well as the results, coming from the students' evaluation, were encouraging. Conclusions: Concerning the theme - the domain ontology - the alternative methodological proposal allowed the students not only to grasp the concepts involved in the Problem-Based Learning approach, but also had an information product outcome: the ontology itself.

\section{Keywords}

Problem-based learning. Teaching methodology. Teachinglearning process. Ontology. Information Systems.

Recebido em 3 de abril de 2013

Aceito em 26 de maio de 2013

\section{Sobre os autores:}

\section{Ricardo Alexandre Afonso}

Bacharel em Análise de Sistemas - FATEB/SP, Especialista em Engenharia de Software - UFSCar/Unilins, Mestre em Ciência da Computação - UFPE, Doutorando em Ciência da Computação - UFPE . afons0055@gmail.com

\section{Clarisse Ferrão Pereira}

Bacharel em Engenharia de Produção - FBV, Mestre em Engenharia Mecânica - UFPE, Aluna especial no Doutorado em Engenharia (Engenharia da Produção) - USP. clarisseferrao@gmail.com

Como citar este artigo:

AFONSO, R. A.; PEREIRA, C. F. MaTUTO: adaptação da metodologia de aprendizagem baseada em problemas aplicada ao ensino de ontologias. AtoZ: novas práticas em informação e conhecimento, Curitiba, v. 2, n. 1, p. 34-43, jan./jun. 2013. Disponivel em: <http://www.atoz.ufpr.br>. Acesso em: 
